\title{
Involvement of glycogen debranching enzyme in bladder cancer (Review)
}

\author{
BENJAMIN WEINHAUS and SUNNY GUIN \\ Gundersen Medical Foundation, La Crosse, WI 54601, USA
}

Received March 3, 2017; Accepted April 7, 2017

DOI: $10.3892 /$ br.2017.907

\begin{abstract}
Bladder cancer is the most common malignancy of the urinary system, however the molecular pathways underlying this disease are incompletely understood. To understand new regulators of bladder cancer progression, the authors carried out a functional genomic screen which identified glycogen debranching enzyme (AGL) as a novel regulator of bladder cancer growth. Glycogen debranching enzyme is involved in glycogen breakdown and germline loss of function mutation of this gene leads to glycogen storage disease type III. To the best of the authors' knowledge, the present study is the first to demonstrate that loss of AGL leads to aggressive bladder tumor growth. AGL mRNA and protein expression in bladder tumors serve as a prognostic marker for patients. Interestingly, AGL's participation in regulating tumor growth is independent of its enzymatic function and involvement with glycogen metabolism in general. Detailed metabolomics and transcriptomic analysis indicated that increases in glucose metabolism, glycine synthesis driven by serine hydroxymethyltransferase 2 and increases in hyaluronic acid synthase 2-driven HA synthesis are major contributors of aggressive bladder tumor growth with loss of AGL. However, the detailed mechanism of how AGL regulates the above mentioned metabolic and genetic pathways is unknown and is being investigated. The present review focuses on AGL's involvement in bladder cancer.
\end{abstract}

\section{Contents}

1. Introduction

2. Identification of glycogen debranching enzyme (AGL) as a new player in bladder cancer

3. AGL, glycogen metabolism and regulation of glycine synthesis

Correspondence to: Dr Sunny Guin, Gundersen Medical Foundation, 1300 Badger St., La Crosse, WI 54601, USA

E-mail: sguin@gundersenhealth.org

Key words: bladder cancer, glycogen debranching enzyme, SHMT2, HAS2, hyaluronic acid, CD44, RHAMM
4. AGL and hyaluronic acid synthesis

5. AGL and hyaluronic acid receptors

6. Conclusion

\section{Introduction}

New medical treatments in oncology depend upon identifying novel prognostic markers and therapeutic targets. Well-studied malignancies, such as breast cancer, have defined mechanisms based on presence or absence of estrogen or progesterone receptors, which can be used as genetic prognostic markers to determine treatment methods $(1,2)$. Non-small cell lung cancer treatments have developed therapies catering towards patients' unique genetic mutations in proto-oncogenes, such as anaplastic lymphoma kinase or in epidermal growth factor receptor genes (3-5). However, molecular pathways and genetic alterations in bladder cancer need to be studied further to have effective treatment options as well. Urinary system tumors are the 5th most common tumor type in the United States, with bladder cancer comprising 54\% of new urinary system-based tumor cases (6). Bladder cancer mortality rates have remained relatively unchanged over the past 25 years, while treatment options with low efficacy are in continued use $(7,8)$. More biological data must be collected to develop proper therapeutic targets and prognostic markers for bladder cancer to improve treatment methods and patient mortality.

\section{Identification of glycogen debranching enzyme (AGL) as a new player in bladder cancer}

To identify new genes responsible for aggressive bladder cancer growth, an in vivo RNA interference library screening was conducted using a whole genome shRNA library (9). This screen identified amylo- $\alpha$-1,6-glucosidase,4- $\alpha$-glucanotrans ferase (AGL) as a potential new regulator of bladder cancer growth (9).

AGL, or glycogen debranching enzyme, is involved in glycogen breakdown (glycogenolysis) (10). Germline mutations identified in AGL have been associated with Cori disease, otherwise known as glycogen storage disease III (GSD III), characterized by irregular glycogenolysis and accumulation of abnormally branched glycogen (limit dextrin) in the liver $(10,11)$. The symptomatic diagnosis of GSD III 
primarily includes poor liver function and, in some cases, poor skeletal muscle function (11). Despite the understanding of AGL in both glycogenolysis and in GSD III, the present authors were the first to report a connection between AGL and bladder cancer $(9,12)$. Analysis of bladder tumors from patients demonstrated that loss of AGL expression (gene and protein) in cancer has been associated with clinically aggressive tumor prognosis and poor patient mortality (9). These studies have developed investigative interest in characterizing AGL loss in bladder cancer.

\section{AGL, glycogen metabolism and regulation of glycine synthesis}

Following identifying AGL as a potential regulator of bladder cancer growth from the functional genomic screen, and demonstrating that loss of AGL expression in bladder tumors leads to poor patient outcome, the authors carried out various cell based functional assays to validate whether AGL serves any functional role in bladder cancer (9). Tumor cells expressing low levels of AGL displayed enhanced anchorage independent and dependent growth, while AGL overexpression decreased anchorage independent and dependent growth (9). Further analysis of AGL low tumor cells presented increased production of limit dextrin, or abnormally branched glycogen, similar to patients with GSD III. To understand in detail whether a connection between inhibition of glycogen breakdown and aggressive bladder cancer growth existed, the authors investigated the role of glycogen phosphorylase (PYG), the other enzyme known to interplay with AGL in glycogen breakdown $(9,10,12)$. In addition, experiments using enzymatic null mutants of AGL were conducted to understand AGL's enzymatic activity in bladder tumor growth (9). Results from these experiments indicated that depletion of PYG did not affect anchorage independent growth of bladder cancer, nor did alteration of the AGL's enzymatic region involved in glycogenolysis have any impact on bladder tumor growth (9). Thus, it was confirmed that neither AGL's known enzymatic function, nor inhibition of glycogen breakdown, serves a role in bladder cancer progression. This confirmed that AGL plays a role in bladder cancer via an unknown mechanism.

Furthermore, low AGL expression was observed to make bladder cancer cells dependent upon higher levels of glucose for growth. To obtain an insight as to how glucose may be involved in aggressive bladder cancer growth with AGL loss, a metabolomics screen was carried out using 13C-labeled glucose and observed for intake and distribution of glucose (9). This study indicated that low AGL expressing cells rely upon glucose to help drive increased glycine synthesis (9). This evidence was supported by the amplified serine hydroxymethyltransferase 2 (SHMT2) gene, responsible for glycine synthesis $(13,14)$. In addition, elevated expression of SHMT2 correlates with poor patient prognosis in bladder cancer patients with low AGL expression, verifying the importance of glycine synthesis in AGL low bladder cancer (9). These findings concluded AGL's biologically relevant position as a tumor suppressor, independent of its enzymatic function, and the potential it has for clinical studies in bladder cancer.

\section{AGL and hyaluronic acid synthesis}

The significance of AGL in bladder cancer is clear, but the mechanisms behind tumor growth in AGL low malignancies require further investigation. To observe genetic differences in AGL low bladder cancer, a transcriptional profiling of human bladder cancer cells with and without AGL expression was performed, searching for genes differentially regulated with loss of AGL (15). Hyaluronic acid synthase 2 (HAS2), a gene associated with HA synthesis $(16,17)$, was one of the overexpressed genes with AGL loss (15). Elevated HAS2 expression is correlated with high-grade bladder cancer and poor patient mortality, making HAS2 a gene of interest for further study in the AGL model of bladder cancer (15).

Hylauronic acid synthases (HAS) are responsible for making hyaluronan, or HA (17). Known as non-branching glycosaminoglycans, HA vary in size based on the number of disaccharide repeats, with glucose serving as the precursor molecule for HA synthesis $(16,17)$. Located in the extracellular matrix, HA can bind to cell surface receptors designed for different sized HA (18). HA is biologically relevant in angiogenesis, anchorage independent growth and for allowing cell-to-cell interactions with cancer tissue and surrounding stromal tissue $(16,19)$. The significance of HA in cancer biology provided a reason to further investigate HAS2-mediated HA synthesis in AGL low bladder cancer.

HAS2 was identified as the HAS isoform of interest, as the expression of other isoforms, HAS1 or HAS3, were found to be irrelevant in AGL depleted cells (15). In concordance with increased expression of HAS2, bladder cancer cells with AGL loss presented increased levels of HA synthesis (15). To further validate the significance of HAS2-driven HA synthesis in AGL low bladder cancer, genetic knockdown of the HAS2 gene and treatment with HA synthesis inhibitor, 4-methylumbelliferone (4MU) (20), was carried out in bladder cancer cells with and without AGL followed by in vitro and in vivo growth assays. Knockdown of HAS2 resulted in loss of anchorage-independent growth, reduced proliferation and xenograft tumor growth of bladder cancer cells without AGL expression (15). Treatment with 4MU specifically inhibited growth of AGL low bladder cancer cells, similar to genetic inhibition of HAS2, in in vitro and xenograft growth assays (15). Further analysis of bladder cancer patient datasets demonstrated that patients with high HAS2 and low AGL mRNA expression exhibit poor outcome, giving credence that bladder tumors with low AGL expression are dependent of HAS2 for aggressive growth (15). These experiments illustrate the importance of HAS2-mediated HA synthesis in bladder carcinoma cells with AGL loss, and provide a potential mechanism of action to investigate for prognostic markers or therapeutic targets.

\section{AGL and hyaluronic acid receptors}

The authors have established that aggressive growth of low AGL expressing bladder cancer relies upon HAS2-driven HA synthesis. However, the downstream signaling triggered by HA in AGL low bladder tumors remains unclear. Hyaladherins, or proteins that bind to extracellular HA, can be either cell surface receptors or extracellular matrix proteins (21). Two of these cell surface hyaladherins, RHAMM and CD44, are 
clinically significant in various cancer models $(16,19,22)$. These hyaladherins have been demonstrated to provide multi-drug resistance, assist with anchorage independent growth, and cell motility of cancer cells (23-25). Since both RHAMM and CD44 serve a major role in tumor progression, the authors investigated whether these proteins were involved in HA driven rapid growth of bladder cancer cells with AGL loss.

RHAMM and CD44 expressed by cancer cells can be changed by manipulating the amount of HA the cancer cells are exposed to $(26,27)$. This has been used as a measure of HA signaling by these receptors. Analysis of CD44 and RHAMM expression in AGL low bladder cancer cells indicated that neither knockdown of HAS2, nor treatment with HA synthesis inhibitor 4MU, was able to reduce expression of CD44 or RHAMM (28). The addition of superfluous HA also made little change to RHAMM and CD44 expression (28). This suggested that changes in HA levels do not affect RHAMM or CD44 expression in AGL low bladder tumors. However, it was shown that genetic inhibition of HAS2-, CD44- or RHAMM-induced apoptosis in AGL low bladder cancer cells, where as they had minimal effect on apoptosis of bladder cancer cells expressing AGL (28). Apoptosis was measured by analyzing caspase signaling and by TUNEL assays analyzing the number of cells undergoing apoptosis (28). The percentage of AGL low bladder cancer cells undergoing apoptosis with knockdown of HAS2, CD44 or RHAMM was similar, suggesting they are part of the same pathway. Moreover, loss of CD44 or RHAMM reduced expression of HAS2 and HA synthesis in AGL low bladder cancer cells, validating that CD44 and RHAMM interaction with HA and signaling is relevant in AGL low bladder tumors (28). Interestingly, a bladder cancer cell line with AGL loss is either dependent on loss of CD44 (UMUC3) or RHAMM (T24T, MGHU4) for induction of apoptosis (28). The reason for cell line based preference for one receptor over the other is unknown. Loss of CD44 and RHAMM predominantly inhibited anchorage dependent and independent growth of AGL low bladder cancer cells as also reported with loss of HAS2 (28). The current research clearly demonstrates that AGL loss promotes aggressive growth of bladder cancer through downstream signaling of hyaladherins CD44 and RHAMM driven by HAS2-mediated HA synthesis.

Analysis of CD44 and RHAMM mRNA expression as a prognostic marker for bladder cancer patients demonstrated that RHAMM alone and in combination with AGL is prognostic of poor bladder cancer patient outcome, while CD44 mRNA was not prognostic in the bladder cancer patient datasets analyzed by us (28). Bladder cancer patients with high RHAMM and low AGL mRNA expression were demonstrated to present poor outcome compared to other groups (28). Thus, bladder cancer patients with low tumor AGL expression and high RHAMM expression can be subjected to personalized treatment with inhibition of HA synthesis or HA signaling driven by RHAMM.

\section{Conclusion}

Through extensive experimentation, the authors have reported that the loss of AGL results in aggressive growth of bladder

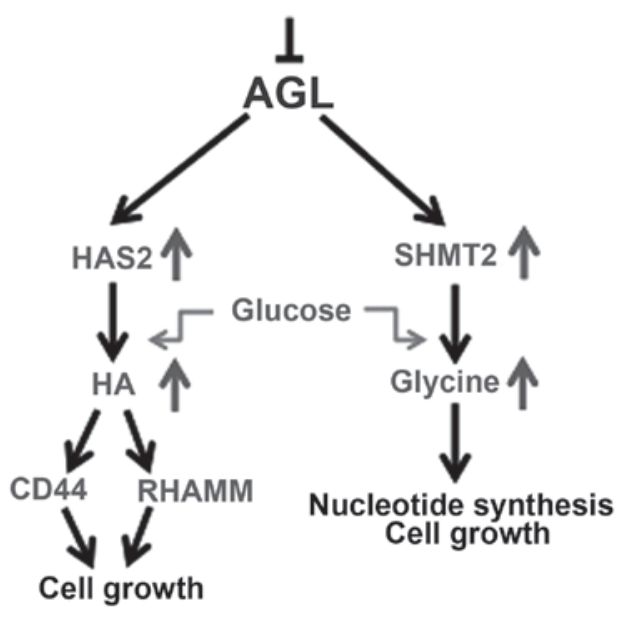

Figure 1. Signaling pathways affected by loss of AGL expression resulting in aggressive growth of bladder tumors. Loss of AGL leads to overexpression of SHMT2 and HAS2, which results in increased glycine and HA synthesis, respectively. This increase in glycine and HA synthesis is supported by increased glucose uptake and metabolism by these cells. Increased glycine synthesis promotes rapid cell proliferation by increasing nucleotide synthesis and increase in HA synthesis promotes tumor growth by signaling through its receptors, CD44 and RHAMM. AGL, glycogen debranching enzyme; SHMT2, hydroxymethyltransferase 2 ; HAS2, hyaluronic acid synthase 2; RHAMM, hyaluronan mediated motility receptor.

cancer cells and AGL mRNA and protein expression serves as a prognostic marker for bladder cancer patients (9). In addition, the loss of AGL, SHMT2-driven glycine synthesis and HAS2-driven HA synthesis serves a major role in driving bladder tumor growth $(9,15,28)$. It has been concluded that glucose is a major driver of glycine synthesis with AGL loss (9), and the authors have unpublished data indicating that glucose is also the major driver of HA synthesis in AGL low bladder cancer cells. Thus, it is believed that the increase in SHMT2 and HAS2 expression with AGL loss drives increased glucose uptake by cells to make more glycine and HA which promotes rapid growth of these cells (Fig. 1).

Very little is known about AGL biology, other than its involvement in glycogen metabolism. It is unknown how HAS2 and SHMT2 levels increase as a response to low AGL expression. AGL could interact with these genes directly, or interact with intermediary biomolecules, which results in overexpression of SHMT2 and HAS2 with loss of AGL in bladder cancer cells. Experiments need to be carried out to understand how AGL regulates the expression of SHMT2, HAS2 and other genes or pathways that influence tumor growth. It is important to investigate and understand which biomolecules interact directly with AGL and are responsible for regulating tumor growth. Understanding how AGL functions as a tumor suppressor, and the different mechanisms of action by which it regulates aggressive bladder cancer growth, would be beneficial in the future for identification of better therapeutic options based of AGL expression status.

\section{Acknowledgements}

The present study was supported by the Bladder Cancer Advocacy Network (BCAN) Young Investigator Award to S.G., and by the Gundersen Medical Foundation. 


\section{References}

1. Bauer KR, Brown M, Cress RD, Parise CA and Caggiano V: Descriptive analysis of estrogen receptor (ER)-negative, progesterone receptor (PR)-negative, and HER2-negative invasive breast cancer, the so-called triple-negative phenotype: A population-based study from the California cancer Registry. Cancer 109: 1721-1728, 2007.

2. Ariazi EA, Ariazi JL, Cordera F and Jordan VC: Estrogen receptors as therapeutic targets in breast cancer. Curr Top Med Chem 6: 181-202, 2006.

3. Waqar SN and Morgensztern D: Precision medicine in lung cancer: The battle continues. J Thorac Dis 8: 2991-2993, 2016.

4. Zhou C, Wu YL, Chen G, Feng J, Liu XQ, Wang C, Zhang S, Wang J, Zhou S, Ren S, et al: Erlotinib versus chemotherapy as first-line treatment for patients with advanced EGFR mutation-positive non-small-cell lung cancer (OPTIMAL, CTONG-0802): A multicentre, open-label, randomised, phase 3 study. Lancet Oncol 12: 735-742, 2011.

5. Solomon BJ, Mok T, Kim DW, Wu YL, Nakagawa K, Mekhail T, Felip E, Cappuzzo F, Paolini J, Usari T, et al; PROFILE 1014 Investigators: First-line crizotinib versus chemotherapy in ALK-positive lung cancer. N Engl J Med 371: 2167-2177, 2014.

6. Siegel RL, Miller KD and Jemal A: Cancer Statistics, 2017. CA Cancer J Clin 67: 7-30, 2017.

7. Zhuo C, Li X, Zhuang H, Tian S, Cui H, Jiang R, Liu C, Tao R and Lin $\mathrm{X}$ : Evaluating the efficacy and safety of intravesical chemotherapies for non-muscle invasive bladder cancer: A network meta-analysis. Oncotarget 7: 82567-82579, 2016.

8. National Cancer Institute: A Snapshot of Bladder Cancer. https:// www.cancer.gov/research/progress/snapshots/bladder. Accessed November 5, 2014.

9. Guin S, Pollard C, Ru Y, Ritterson Lew C, Duex JE, Dancik G, Owens C, Spencer A, Knight S and Holemon H: Role in tumor growth of a glycogen debranching enzyme lost in glycogen storage disease. J Natl Cancer Inst 106: pii: dju062, 2014.

10. Adeva-Andany MM, González-Lucán M, Donapetry-García C, Fernández-Fernández C and Ameneiros-Rodríguez E: Glycogen metabolism in humans. BBA Clin 5: 85-100, 2016.

11. Dagli A, Sentner CP and Weinstein DA: Glycogen Storage Disease Type III. In: GeneReviews ${ }^{\circledR}$ [Internet]. Pagon RA, Adam MP, Ardinger HH, et al (eds). University of Washington, Seattle, WA, 1993-2017.

12. Ritterson Lew C, Guin S and Theodorescu D: Targeting glycogen metabolism in bladder cancer. Nat Rev Urol 12: 383-391, 2015.

13. Hebbring SJ, Chai Y, Ji Y, Abo RP, Jenkins GD, Fridley B, Zhang J, Eckloff BW, Wieben ED and Weinshilboum RM: Serine hydroxymethyltransferase 1 and 2: Gene sequence variation and functional genomic characterization. J Neurochem 120: 881-890, 2012.

14. Jain M, Nilsson R, Sharma S, Madhusudhan N, Kitami T, Souza AL, Kafri R, Kirschner MW, Clish CB and Mootha VK: Metabolite profiling identifies a key role for glycine in rapid cancer cell proliferation. Science 336: 1040-1044, 2012.
15. Guin S, Ru Y, Agarwal N, Lew CR, Owens C, Comi GP and Theodorescu D: Loss of Glycogen Debranching Enzyme AGL Drives Bladder Tumor Growth via Induction of Hyaluronic Acid Synthesis. Clin Cancer Res 22: 1274-1283, 2016.

16. Karbownik MS and Nowak JZ: Hyaluronan: Towards novel anti-cancer therapeutics. Pharmacol Rep 65: 1056-1074, 2013.

17. Tammi RH, Passi AG, Rilla K, Karousou E, Vigetti D, Makkonen K and Tammi MI: Transcriptional and post-translational regulation of hyaluronan synthesis. FEBS J 278: 1419-1428, 2011.

18. Wang A, de la Motte C, Lauer M and Hascall V: Hyaluronan matrices in pathobiological processes. FEBS J 278: 1412-1418, 2011.

19. Lokeshwar VB, Mirza S and Jordan A: Targeting hyaluronic acid family for cancer chemoprevention and therapy. Adv Cancer Res 123: 35-65, 2014.

20. Nagy N, Kuipers HF, Frymoyer AR, Ishak HD, Bollyky JB, Wight TN and Bollyky PL: 4-methylumbelliferone treatment and hyaluronan inhibition as a therapeutic strategy in inflammation, autoimmunity, and cancer. Front Immunol 6: 123, 2015

21. Nikitovic D, Kouvidi K, Kavasi RM, Berdiaki A and Tzanakakis GN: Hyaluronan/Hyaladherins - a Promising Axis for Targeted Drug Delivery in Cancer. Curr Drug Deliv 13: 500-511, 2016.

22. Dicker KT, Gurski LA, Pradhan-Bhatt S, Witt RL, Farach-Carson MC and Jia X: Hyaluronan: A simple polysaccharide with diverse biological functions. Acta Biomater 10: $1558-1570,2014$

23. Orian-Rousseau V: CD44 Acts as a Signaling Platform Controlling Tumor Progression and Metastasis. Front Immunol 6: 154, 2015.

24. Misra S, Hascall VC, Markwald RR and Ghatak S: Interactions between Hyaluronan and Its Receptors (CD44, RHAMM) Regulate the Activities of Inflammation and Cancer. Front Immunol 6: 201, 2015.

25. Maxwell CA, McCarthy J and Turley E: Cell-surface and mitotic-spindle RHAMM: Moonlighting or dual oncogenic functions? J Cell Sci 121: 925-932, 2008.

26. Lokeshwar VB, Lopez LE, Munoz D, Chi A, Shirodkar SP, Lokeshwar SD, Escudero DO, Dhir N and Altman N: Antitumor activity of hyaluronic acid synthesis inhibitor 4-methylumbelliferone in prostate cancer cells. Cancer Res 70: 2613-2623, 2010.

27. Yates TJ, Lopez LE, Lokeshwar SD, Ortiz N, Kallifatidis G, Jordan A, Hoye K, Altman N and Lokeshwar VB: Dietary supplement 4-methylumbelliferone: an effective chemopreventive and therapeutic agent for prostate cancer. J Natl Cancer Inst 107: djv085, 2015.

28. Oldenburg D, Ru Y, Weinhaus B, Cash S, Theodorescu D and Guin S: CD44 and RHAMM are essential for rapid growth of bladder cancer driven by loss of Glycogen Debranching Enzyme (AGL). BMC Cancer 16: 713, 2016. 